\title{
Perioperative Care of a Pediatric Patient With Cockayne Syndrome
}

\author{
Asad A. Khawaja ${ }^{a}$, Joseph D. Tobias ${ }^{\text {b, c, d }}$
}

\begin{abstract}
First described in 1936, Cockayne is an autosomal recessive neurodegenerative disorder characterized by dwarfism, premature aging, impaired development of the nervous system, dental complications, abnormal photosensitivity, and optic atrophy. Of primary concern to anesthesia providers is the potential for a difficult airway related to many physical features including micrognathia, a small mouth, large teeth, overbite, limited mouth opening, and restriction of temporomandibular joint movement. Additional comorbid involvement includes associated coronary artery disease, hypertension, and diabetes. The perioperative implications of Cockayne syndrome are discussed, previous perioperative reports are reviewed, and options for anesthetic care are presented.
\end{abstract}

Keywords: Cockayne syndrome; Anesthesia

\section{Introduction}

Described in 1936, Cockayne syndrome (CS), also known as Neill-Dingwall syndrome, is a rare autosomal recessive neurodegenerative disorder characterized by dwarfism, premature aging, impaired development of the nervous system, dental complications, abnormal photosensitivity, and optic atrophy [1, 2]. Confirmation of the diagnosis is possible using genetic testing with the demonstration of a repair/synthesis defect of DNA/ RNA in response to ultra-violet radiation in cultured fibroblast cells $[3,4]$. The defective DNA repair from mutations in the excision repair cross-complementation group 6 (ERCC6) and ERCC8 may be a cause of Alzheimer neurofibrillary tangles as well as lipofuscin accumulation in neurons and calcific vascu-

Manuscript submitted September 11, 2017, accepted September 20, 2017

${ }^{a}$ Marshall University, Joan C. Edwards School of Medicine, Huntington, WV, USA

bDepartment of Anesthesiology and Pain Medicine, Nationwide Children's Hospital, Columbus, OH, USA

'Department of Anesthesiology and Pain Medicine, The Ohio State University, Columbus, OH, USA

${ }^{\mathrm{d}}$ Corresponding Author: Joseph D. Tobias, Department of Anesthesiology and Pain Medicine, Nationwide Children's Hospital, 700 Children's Drive, Columbus, OH 43205, USA. Email: Joseph.Tobias@Nationwidechildrens.org

doi: https://doi.org/10.14740/jmc2913w lopathy which contribute to the impaired development of the central nervous system (CNS) and premature aging $[4,5]$. At birth, head circumference may not be normal, but CNS growth failure results in progressive microcephaly [6]. Intracranial calcifications and dysfunction of the CNS including the basal ganglia results in cerebellar ataxia, mental retardation, and blindness [1]. The phenotypic pattern is consistent with an abnormal facies with a beak-like pointed nose, large ears, micrognathia, and a loss of subcutaneous fat around the cheeks resulting in what has been described as a "bird-like" appearance.

Additional clinical manifestations include growth retardation with failure to thrive, premature aging, type 2 diabetes mellitus, cardiovascular involvement, osteopenia, kyphosis, hair thinning, and skin atrophy [7]. Growth failure due to disproportionate dwarfism and ataxia results in a gait disorder with the majority of patients becoming non-ambulatory [1, 8-10]. Life expectancy is approximately $10-12$ years of age, especially in patients with the early onset type of CS (see below). Given the multi-system involvement of the disorder, patients may present for surgical procedures of the airway, CNS, or the heart. We present a 5-year-old boy with CS who presented for bilateral club foot repair. The perioperative concerns of such patients are discussed and previous reports of anesthetic care are reviewed.

\section{Case Report}

Patient or parent consent for publication of a case report is not required by the Institutional Review Board of Nationwide Children's Hospital (Columbus, OH, USA). This patient was cared for during a surgical mission trip of Kids First Orthopedic Group (Nashville, TN) to San Miguel, Mexico. The patient was a $9.6 \mathrm{~kg}$, 5-year-old child with CS who presented for bilateral club foot repair. CS was diagnosed during the first year of life during an evaluation for poor weight gain and abnormal facies. The patient was on no medications. There was no significant past medical or surgical history. On physical examination, the patient was noted to have microcephaly and an abnormal facies with a beak-like pointed nose, large ears, and micrognathia. He was non-verbal and ambulated only with a walker or support. Airway examination was difficult due to lack of cooperation, but revealed a Mallampati class 3 airway. The cardiovascular and respiratory examinations were unremarkable. Preoperative vital signs include heart rate (HR) of 118 beats/ min, blood pressure (BP) of $84 / 46 \mathrm{~mm} \mathrm{Hg}$, respiratory rate of 20 breaths/min, body temperature of $36{ }^{\circ} \mathrm{C}$, and room air 
Table 1. Summary of Previous Reports of Anesthetic Care for Patients With Cockayne Syndrome

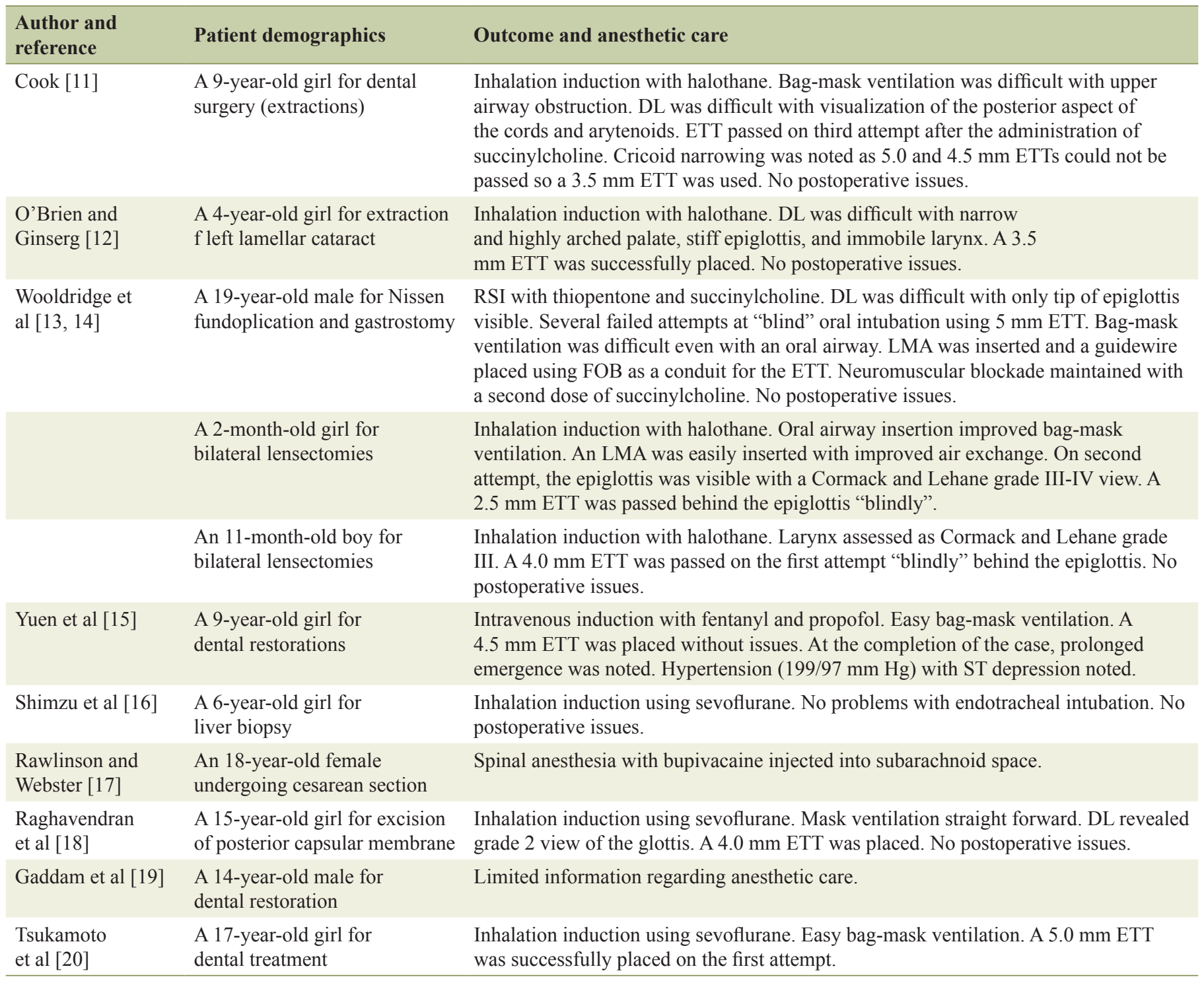

DL: direct laryngoscopy; ETT: endotracheal tube; RSI: rapid sequence intubation; LMA: laryngeal mask airway; FOB: fiberoptic bronchoscope.

oxygen saturation of $96 \%$. The patient was held nil per os for $6 \mathrm{~h}$ for solids and $2 \mathrm{~h}$ for clear liquids. He was transported to the operating room and standard American Society of Anesthesiologists' monitors were placed. Anesthesia was induced by the inhalation of sevoflurane in oxygen and a peripheral intravenous catheter was placed. Bag-valve-mask ventilation was provided without difficulty. Propofol $(1.5 \mathrm{mg} / \mathrm{kg})$ was administered and a size 2 laryngeal mask airway (LMA) was placed. Following LMA placement, the patient was placed in the lateral Sims' position and a caudal epidural block was placed with the administration of $10 \mathrm{~mL}$ of $0.25 \%$ bupivacaine with epinephrine 1:200,000 and clonidine $(10 \mu \mathrm{g})$. Anesthesia was maintained with 2-3\% sevoflurane (inspired concentration) in oxygen with spontaneous ventilation and fentanyl $(1 \mu \mathrm{g} / \mathrm{kg})$. No response was noted to surgical incision and the sevoflurane was decreased to $1.5-2 \%$. Intraoperative vital signs were sta- ble with an HR of 110 - 140 beats/min, BP of $68-88 / 40-58$ $\mathrm{mm} \mathrm{Hg}$, oxygen saturation of $97-100 \%$, and respiratory rate of 18 - 24 breaths/min. The operative procedure lasted $75 \mathrm{~min}$. Total intraoperative blood loss was less than $10 \mathrm{~mL}$. Intraoperative fluids included $220 \mathrm{~mL}$ of Hartmann's solution. At the completion of the surgical procedure, the LMA was removed and the patient was transported to the post-anesthesia care unit (PACU). His postoperative course was unremarkable. He was discharged to the inpatient ward from the PACU and then home the following day. Postoperative analgesia was provided by intermittent doses of oral acetaminophen.

\section{Discussion}

There are several perioperative concerns to consider when 
planning anesthetic care for patients with CS. Previous reports of anesthetic care for patients with CS are summarized in Table 1 [11-20]. As with any anesthetic care, appropriate preoperative preparation begins with a thorough history and physical examination. Of primary concern to anesthesia providers is the potential for a difficult airway related to many physical features including micrognathia, a small mouth, large teeth, overbite, limited mouth opening, and restriction of temporomandibular joint movement. The available literature clearly shows that direct laryngoscopy may be problematic and that bag-valve-mask ventilation may be difficult. In most cases of difficult bag-valve-mask ventilation, the placement of an LMA has resulted in effective gas exchange. Given these concerns, the appropriate equipment for dealing with the difficult airway including indirect video-laryngoscopy should be readily available prior to anesthetic induction [21]. General anesthesia can be induced by the inhalation of sevoflurane in $100 \%$ oxygen with the maintenance of spontaneous ventilation. The report of Tsukamoto et al suggests that the use of continuous processed electroecncephalogram (EEG) monitoring with devices such as the bispectral index (BIS) may be useful as there may be discordance between the hemodynamic parameters and the depth of anesthesia from the EEG [20]. In their report, the authors noted a rapid decline of the BIS to less than 10 during the induction of anesthesia with $8 \%$ sevoflurane. The administration of neuromuscular blocking agents (NMBAs) should be avoided until adequate bag-valve-mask ventilation is demonstrated. Due to degeneration and involvement of the CNS, patients may have associated gastroesophageal reflux, suggesting that an RSI is indicated. However, given for the potential for airway difficulties, inhalation induction with the application of cricoid pressure may be preferable to RSI. The available anecdotal evidence has demonstrated that in many cases, a smaller than age-appropriate endotracheal tube (ETT) is frequently required due to the associated growth retardation and the potential for subglottic narrowing of the airway.

With the associated neurologic and muscle involvement, one of the main perioperative concerns in providing anesthetic care of such patients is the choice of NMBA. Although it provides a rapid onset of neuromuscular blockade and is often chosen for RSI or if difficulties with endotracheal intubation are anticipated, it has been suggested that succinylcholine be avoided in CS due to the associated neurologic and myopathic involvement and the potential for an exaggerated hyperkalemic response $[22,23]$. In our patient, given the surgical procedure, NMBAs were not required and the airway was managed with an LMA and spontaneous ventilation was maintained. In the event that neuromuscular blockade is required for the surgical procedure, non-depolarizing NMBAs (vecuronium or atracurium) should be used with care as the effect may be prolonged even with routine dosing in patients with pre-existing neuromuscular diseases or hypotonia. Alternatively, where available, suggamadex may provide an additional margin of safety for reversal of the neuromuscular blocking effects of rocuronium or vecuronium.

Comorbid end-organ involvement with CS may also include cardiovascular, endocrine, and renal involvement. Hypertension commonly occurs as part of CS, with elevated renin levels [24]. Autopsy data have demonstrated premature cer- ebral arteriosclerosis with the potential for strokes [2, 25, 26]. Coronary artery involvement may predispose to myocardial ischemia as demonstrated by one case report of Yuen et al [15]. During perioperative care, ECG monitoring for ischemia with control of factors regulating myocardial oxygen delivery including diastolic BP and heart rate is suggested. Diabetes mellitus and renal involvement have been reported with CS. Renal biopsy specimens have revealed collapsed glomeruli with a thickened basement membrane, atrophic capillary loops, and advanced hyalinization resulting in decreased renal function [27]. As indicated, preoperative evaluation of renal function, electrolytes, and blood glucose may be indicated.

In summary, we present the anesthetic considerations of a 5-year-old boy with $\mathrm{CS}$, an autosomal recessive neurodegenerative disorder characterized by dwarfism, premature aging, impaired development of the nervous system, dental complications, abnormal photosensitivity, and optic atrophy. Primary perioperative concerns include the potential for difficulties with airway management including bag-valve-mask ventilation and endotracheal intubation. Various physical features may result in problematic airway management including micrognathia, a small mouth, large teeth, overbite, limited mouth opening, and restriction of temporomandibular joint movement. Additional end-organ involvement includes progressive CNS degeneration with mental retardation, cardiovascular involvement with hypertension and accelerated atherosclerotic disease with myocardial ischemia, diabetes mellitus, and renal insufficiency. The preoperative assessment of end-organ impairment by the primary disease process, preparation for potential difficulties with endotracheal intubation, and close postoperative monitoring are suggested for the effective perioperative care of these patients.

\section{References}

1. Cockayne EA. Dwarfism with retinal atrophy and deafness. Arch Dis Child. 1936;11(61):1-8.

2. Paddison RM, Moossy J, Derbes VJ, Kloepfer W. Cockayne's syndrome. a report of five new cases with biochemical, chromosomal, dermatologic, genetic and neuropathologic observations. Dermatol Trop Ecol Geogr. 1963; 15:195-203.

3. Cleaver JE, Lam ET, Revet I. Disorders of nucleotide excision repair: the genetic and molecular basis of heterogeneity. Nat Rev Genet. 2009;10(11):756-768.

4. He C, Sun M, Wang G, Yang Y, Yao L, Wu Y. Two novel mutations in ERCC6 cause Cockayne syndrome B in a Chinese family. Mol Med Rep. 2017;15(6):3957-3962.

5. Soffer D, Grotsky HW, Rapin I, Suzuki K. Cockayne syndrome: unusual neuropathological findings and review of the literature. Ann Neurol. 1979;6(4):340-348.

6. Rapin I, Lindenbaum Y, Dickson DW, Kraemer KH, Robbins JH. Cockayne syndrome and xeroderma pigmentosum: DNA repair disorders with overlaps and paradoxes. Neurology. 2000;55:1442-1449.

7. Rapin I, Weidenheim K, Lindenbaum Y, Rosenbaum P, Merchant SN, Krishna S, Dickson DW. Cockayne syndrome in adults: review with clinical and pathologic study of a new case. J Child Neurol. 2006;21(11):991-1006. 
8. Nance MA, Berry SA. Cockayne syndrome: review of 140 cases. Am J Med Genet. 1992;42(1):68-84.

9. Natale V. A comprehensive description of the severity groups in Cockayne syndrome. Am J Med Genet A. 2011;155A(5):1081-1095.

10. Mallery DL, Tanganelli B, Colella S, Steingrimsdottir H, van Gool AJ, Troelstra C, Stefanini M, et al. Molecular analysis of mutations in the CSB (ERCC6) gene in patients with Cockayne syndrome. Am J Hum Genet. 1998;62(1):77-85.

11. Cook S. Cockayne's syndrome. Another cause of difficult intubation. Anaesthesia. 1982;37(11):1104-1107.

12. O'Brien FC, Ginsberg B. Cockayne syndrome: a case report. AANA J. 1994;62(4):346-348.

13. Wooldridge WJ, Dearlove OR. Anaesthesia for Cockayne's syndrome: Contemporary solutions to an old problem. Paediatr Anaesth. 1994;4:191-195.

14. Wooldridge WJ, Dearlove OR, Khan AA. Anaesthesia for Cockayne syndrome. Three case reports. Anaesthesia. 1996;51(5):478-481.

15. Yuen MK, Rodrigo MR, Law Min JC, Tong CK. Myocardial ischemia and delayed recovery after anesthesia in a patient with Cockayne syndrome: a case report. J Oral Maxillofac Surg. 2001;59(12):1488-1491.

16. Shimizu T, Inomata S, Miyabe M, Toyooka H. [Anesthesia for a patient with Cockayne's syndrome]. Masui. 2002;51(1):39-41.

17. Rawlinson SC, Webster VJ. Spinal anaesthesia for caesarean section in a patient with Cockayne syndrome. Int J Obstet Anesth. 2003;12(4):297-299.

18. Raghavendran S, Brown KA, Buu N. Perioperative management of patients with Cockayne syndrome - recognition of accelerated aging with growth arrest. Paediatr
Anaesth. 2008;18(4):360-361.

19. Gaddam D, Thakur MS, Krothapalli N, Kaniti S. Dental management of a 14-year-old with Cockayne syndrome under general anesthesia. Case Rep Dent. 2014;2014:925258.

20. Tsukamoto M, Hitosugi T, Yokoyama T. Discrepancy between electroencephalography and hemodynamics in a patient with Cockayne syndrome during general anesthesia. J Clin Anesth. 2016;35:424-426.

21. Engelhardt T, Weiss M. A child with a difficult airway: what do I do next? Curr Opin Anaesthesiol. 2012;25(3):326-332.

22. Martyn JA, Richtsfeld M. Succinylcholine-induced hyperkalemia in acquired pathologic states: etiologic factors and molecular mechanisms. Anesthesiology. 2006;104(1):158-169.

23. Kendig JJ, Bunker JP, Endow S. Succinylcholine-induced hyperkalemia: effects of succinylcholine on resting potentials and electrolyte distributions in normal and denervated muscle. Anesthesiology. 1972;36(2):132-137.

24. Higginbottom MC, Griswold WR, Jones KL, Vasquez MD, Mendoza SA, Wilson CB. The Cockayne syndrome: an evaluation of hypertension and studies of renal pathology. Pediatrics. 1979;64(6):929-934.

25. Inoue T, Sano N, Ito Y, Matsuzaki Y, Okauchi Y, Kondo $\mathrm{H}$, Horiuchi N, et al. An adult case of Cockayne syndrome without sclerotic angiopathy. Intern Med. 1997;36(8):565-570.

26. Moossy J. The neuropathology of Cockayne's syndrome. J Neuropathol Exp Neurol. 1967;26(4):654-660.

27. Sato H, Saito T, Kurosawa K, Ootaka T, Furuyama T, Yoshinaga K. Renal lesions in Cockayne's syndrome. Clin Nephrol. 1988;29(4):206-209. 